\title{
PREVALENCIA DE CYBERBULLYING EN JÓVENES ESTUDIANTES COLOMBIANOS HOMOSEXUALES Y BISEXUALES
}

\author{
Camilo Soto Hernández, Oscar Salcedo Lopera, Jesús Redondo Pacheco \\ y Marianela Luzardo Briceño \\ Universidad Pontificia Bolivariana \\ Colombia
}

\begin{abstract}
RESUMEN
La prevalencia del ciberbullying crece en la medida que la tecnología permite el acceso rápido a la comunicación virtual y se hace accesible a la mayoría de personas, trayendo consigo graves efectos en la salud psicológica e incluso física de las víctimas. Las causas por las que se hace son muy variadas; la de interés aquí es por orientación sexual en adolescentes escolarizados, ya sean homosexuales o bisexuales. El objetivo del trabajo reside en determinar la prevalencia de ciberbullying por homofobia en personas con orientación sexual distinta a la heterosexual, en dos colegios de educación media. La investigación fue de corte transversal, de alcance descriptivo. Participaron 192 estudiantes de secundaria de grados octavo y noveno de un colegio público y uno privado de la ciudad de Bucaramanga, Colombia, de los que $53 \%$ fueron mujeres y $47 \%$ hombres. Los resultados indican que en los colegios analizados hay una prevalencia de $27.6 \%$ de este fenómeno social, y que las minorías sexuales (homosexuales y bisexuales) han sido víctimas, victimarios y observadores. Sin embargo, no es posible establecer si el ciberbullying al que estuvieron expuestos fue por razón de su orientación sexual.
\end{abstract}

Palabras Clave:

ciberbullying, homosexualidad, bisexualidad, tipo de colegio, jóvenes

\section{PreVAlENCE OF CYBERBULlyING IN YOUNG hOMOSEXUAL AND BISEXUAL COLOMBIAN STUDENTS}

\begin{abstract}
The prevalence of ciberbullying is growing as technology allows rapid access to virtual communication and is made accessible to most people, bringing serious effects on the psychological and even physical health of the victims. The causes for which it is executed are varied, the one of interest here is for sexual orientation in adolescent students, whether homosexual or bisexual. The main objective of the work is to determine the prevalence of ciberbullying by homophobia in people with sexual orientation other than heterosexual, in two secondary schools. The research was cross-sectional, descriptive in scope. 192 high school students from eighth and ninth grades from a public and private school in the city of Bucaramanga, Colombia participated, of which $53 \%$ were women and $47 \%$ men. The results indicate that in the schools analyzed there is a prevalence of $27.6 \%$ this social phenomenon, and that sexual minorities (homosexuals and bisexuals) have been victims, perpetrators and observers. However, it could not be established whether the ciberbullying to which they were exposed was due to their sexual orientation.
\end{abstract}

Keywords:

ciberbullying, homosexuality, bisexuality, type of school, youth

Bitácora del ARtículo:

| Recibido: 11 de Diciembre de 2018 | Aceptado: 10 de Septiembre de 2019 | Publicado en línea: Enero - Junio de 2020 | 
Artículo empírico | Prevalencia de cyberbullying en jóvenes...| Soto-Hernández, Salcedo-Lopera, Redondo-Pacheco y Luzardo-Briceño

\title{
Autoría y Derechos de Propiedad Intelectual
}

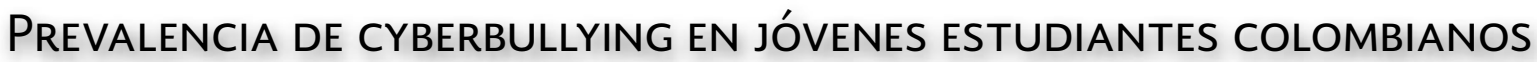 HOMOSEXUALES Y BISEXUALES}

\author{
Camilo Soto Hernández, Oscar Salcedo Lopera, Jesús Redondo Pacheco \\ y Marianela Luzardo Briceño \\ Universidad Pontificia Bolivariana \\ Colombia
}

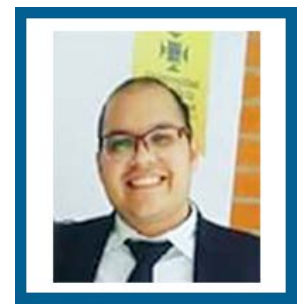

Camilo Soto Hernández

Universidad Pontificia Bolivariana

Correo: camilosotto1@gmail.com

Psicólogo egresado de la Universidad Pontificia Bolivariana. Miembro del Semillero de Investigación Análisis y Transformación Psicosocial SIAPS (2016 - 2018). Agente de Prevención contra el delito de la trata de personas, Fundación CreSer y Oficina de las Naciones Unidas Contra la Droga y el Delito UNODC (2014).

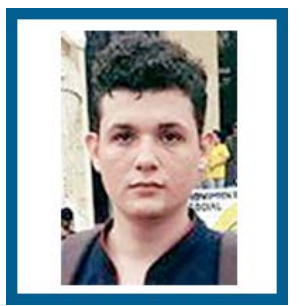

Oscar Salcedo Lopera

Universidad Pontificia Bolivariana

Correo: oesalcedo@outlook.com

Psicólogo egresado de la Universidad Pontificia Bolivariana sede Bucaramanga, Colombia (2018).

\section{CONTRIBUCIÓN DE LOS AUtORES}

Camilo Soto coordinó y contribuyó en todo el proceso metodológico y conceptual. | Oscar Salcedo contribuyó en el proceso conceptual y metodológico. | Jesús Redondo Pacheco revisó y supervisó cada una de las partes del artículo | Marianela Luzardo realizó el análisis estadístico en el programa SPSS.

\section{AGRADECIMIENTOS}

Se agradeces a todas las instuciones y personas que hicieron prosible la realización de está investigación.

\section{DATOS DE Filiación de los AUtores}

Universidad Pontificia Bolivariana

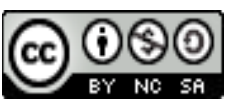

Copyright: ( 2020 Soto-Hernández, C.; Salcedo-Lopera, O.; Redondo-Pacheco y Luzardo-Briceño M

Este es un artículo de acceso abierto distribuido bajo los términos de la licencia Creative Commons Reconocimiento-NoComercial 4.0 Internacional, por lo que su contenido gráfico y escrito se puede compartir, copiar y redistribuir total o parcialmente sin necesidad de permiso expreso de sus autoras con la única condición de que no se puede usar con fines directamente comerciales y los términos legales de cualquier trabajo derivado deben ser los mismos que se expresan en la presente declaración. La única condición es que se cite la fuente con referencia a la Revista Digital Internacional de Psicología y Ciencia Social y a sus autoras. 


\section{TABLA DE CONTENIDO}

Los estudiantes desde una mirada sociocultural, 264

\section{MÉTODO}

Participantes, 265

Materiales, 265

Procedimiento, 265

Análisis de datos, 266

\section{RESULTADOS}

El estudiante universitario como usuario de Facebook, 266

Dilemas para la organización del equipo de trabajo entre los

estudiantes, 267

El trabajo en equipo: Un proceso de negociaciones mediadas por

Facebook, 269

El objetivo. El término del trabajo, 271

Discusión

\section{CONCLUSIONES}

REFERENCIAS 


\section{$\mathbf{L}$} a incursión de las nuevas tecnologías informáticas de comunicación (TIC), como el internet o el teléfono celular, ha cambiado el modo de interacción humana. Las relaciones son inmediatas, rápidas y descomprometidas, revolucionando la manera en que se socializa. Bien sabemos que la socialización es un proceso necesario para la conservación de la ideología social y la transmisión de normas y tradiciones donde las personas establecen vínculos afectivos y emocionales (Simkin y Becerra, 2013). Además, la violencia es un tipo de asociación dinámica que caracteriza las relaciones humanas, susceptible de ser expresadas de múltiples maneras y en escenarios de acuerdo con el rol y a la posibilidad social de los actores, destacando la violencia de género, la violencia política y transpolítica, la violencia interpersonal, la violencia económica, psicológica, sexual, escolar, y la violencia militar, entre otras (Blair, 2009). Así, Rojas (1996, p. 11) define la violencia como el "uso intencionado de la fuerza física en contra de un semejante con el propósito de herir, abusar, robar, humillar, dominar, ultrajar, torturar, destruir o causar la muerte". En este sentido, el ciberbullying se refiere al acoso entre pares por medio de las TIC (García-Maldonado, Joffre-Velázquez, Martínez-Salazar y Llanes-Castillo, 2011; Morales, Rueda, Redondo, Luzardo y Gómez, 2018); en este caso son niños, jóvenes y adolescentes familiarizados con las TIC los que protagonizan esta forma de violencia, aunque es necesario aclarar que los adultos no están exentos a esta problemática (Hernández y Solano, 2007). De esta manera, el ciberbullying es un acto agresivo e intencionado que se efectúa mediante el contacto electrónico contra uno o varios individuos que no tiene intención de defenderse o se siente imposibilitado para ello, convirtiéndose en un acto vejatorio que tiene como objetivo difamar de manera deliberada, repetitiva y hostil (Besley, 2005; Garaigordobil, 2011; Grigg, 2010; Smith, 2000). Los efectos del hostigamiento virtual recaen en el rendimiento académico, el modo de relacionarse con los demás y en otras actividades de su normal desarrollo psicosocial; además, estos efectos se asocian a estados de vulnerabilidad, depresión y ansiedad, e incluso pueden llevar al suicidio (Avilés, 2009).

En este orden de ideas, es preocupante que la práctica del ciberbullying se dé en mayor medida en edades entre los 16 y los 20 años, etapas donde el desarrollo psicológico y la inclusión a la vida social tienen mucha importancia (Lorenzo, 2012), además de ser fundamental el desarrollo de habilidades sociales que le permiten a los niños, jóvenes y adolescentes lograr desenvolverse en cualquier contexto, tanto en su etapa escolar como en la profesional, personal y afectiva. En este panorama el ciberbullying aparece como una amenaza para nativos digitales y como un problema para padres y educadores que aún no conocen las consecuencias de este fenómeno (Mura y Diamantini, 2013).

El estudio del ciberbullying es actual, se trata de un fenómeno en auge por el uso popular de las redes sociales, los correos electrónicos y los teléfonos celulares. En este sentido, el uso de redes sociales aumenta la posibilidad de ser cyber-victimizado (García-Maldonado et al. 2011; Morales et al., 2018; Mura y Diamantini, 2013). No obstante, la transmisión de archivos, envío de correos electrónicos, conversaciones en línea y el uso de celulares como vías para el hostigamiento, hace que la violencia tenga mayor alcance y formas de propagarse. Al respecto, las investigaciones sobre la prevalencia del ciberbullying aumentan a medida que pasa el tiempo (Williams y Guerra, 2007). Por ejemplo, Finkelhor, Mitchell y Wolak (2000) en Estados Unidos con una muestra de 1500 adolescentes no escolarizados entre 10 y 17 años encontró que el 6\% de estos eran victimizado y el $1 \%$ agresor. También en otra investigación en Estados Unidos con una muestra de 100 adolescentes, se encontró que al menos una cuarta parte había publicado algo de lo que se arrepintió porque pares se burlaron; un $25 \%$ había creado identidades falsas en línea y uno de cada diez había publicado un desnudo o semi-desnudo de sí mismo (Tucker, 2009).

Además, Keith y Martin (2005) descubrieron en una muestra de 1566 estudiantes norteamericanos de 9 a 13 años de edad que el $42 \%$ de estos habían estado implicados en ciberbullying. Según Oliveros, Amemiya, Condorimay, Oliveros, Barrientos y Rivas (2012), el acoso es efectuado directamente con videos, imágenes, suplantación de la personalidad, inicio de rumores, exclusión y mensajes de texto o de voz que ridiculizan o ultrajan la integridad psicológica de la víctima.

Si bien las consecuencias negativas del ciberacoso han sido bien documentadas, se ha enfatizado mayormente cómo afecta a las víctimas. Estos efectos incluyen emociones negativas como miedo, ira, indefensión, vergüenza y culpa; problemas emocionales como ansiedad o depresión; mayor riesgo de problemas psicosomáticos; problemas de ajuste psicosocial; mayores problemas de salud mental; bajo rendimiento académico; e incluso, en los casos más extremos, intento de suicidio o suicidio (Arseneault, Bowes y Shakoor, 2010; Espelage, Low, Van Ryzin, y Polanin, 2015; Redondo, Luzardo y Rangel, 2016). 
Sin embargo, la comprensión actual del acoso y el ciberacoso sigue siendo incompleta en ciertas áreas que, debido a su naturaleza estructural, merecen atención específica, como el acoso y el ciberacoso homofóbico, un problema que se considera universal (UNESCO, 2013).

Consiguiente a esto, el concepto de homofobia puede definirse como una actitud hostil y de odio irracional hacia los homosexuales sin importar si son hombres o mujeres, entendida como un estado psicológico que puede ser considerado como enfermedad por su precipitación irracional a la violencia (Cruz, 2002).

En este sentido, en un estudio realizado por Piedra, Rembrandt, Ries y Ramírez (2013) con 245 estudiantes de la Facultad de Ciencias de la Educación de la Universidad de Sevilla, España, se aplicó un cuestionario sobre conductas heterosexistas y homofóbicas percibidas en el periodo de educación secundaria, encontrando un considerable índice de estudiantes que fueron testigos o experimentaron algún tipo de comportamiento hostil. Así, 30.6\% de los encuestados indicaron haber sido testigos de comportamientos homofóbicos muchas veces, mientras que $33.9 \%$ reportaron que en ocasiones habían vivido dichas situaciones. Otro de los datos relevantes de este estudio es que $40 \%$ de los estudiantes dijeron haber padecido en alguna ocasión, de manera directa, comportamientos homofóbicos.

Colombia no es ajena a esta problemática, y aunque son escasos los estudios en el país acerca del acoso escolar por orientación sexual, y que no se ha reconocido la dimensión real del acoso homofóbico (Chaux, 2012; UNESCO, 2013) una línea base desarrollada por la Organización Colombia Diversa para determinar la situación de la población de lesbianas, gais, bisexuales y transgénero (LGBTI), identificó que en la capital colombiana $11.91 \%$ de los jóvenes que dejan de asistir al colegio lo hacen por su orientación sexual o por su identidad de género (Acosta, Cuellar, y Martínez, 2013).

Asimismo, la Personería Municipal de Santiago de Cali (2012) también hizo un estudio exploratorio con 280 estudiantes, identificando que $3.92 \%$ de los sujetos indicaron haber sufrido discriminación por su orientación sexual. Al ser consultados respecto a si tenían conocimientos de compañeros que hubiesen sido discriminados por su orientación sexual, 35.7\% indicaron conocer algunos casos.

Gracias al avance tecnológico hoy se sabe de casos que no son denunciados y que van en aumento (Garaigordobil, 2011; Piedra et al., 2013). En Latinoamérica la discriminación por orientación sexual se ha convertido en uno de los problemas más preocupantes de los sistemas escolares, implementándose iniciativas focalizadas, pero sin alcances a nivel nacional y poco documentadas (Artiles y Kozleski, 2014; Espejo, 2018). A pesar de esto, no se puede desconocer que el problema de este tipo de discriminación y su expresión por medio del bullying y ciberbullying homofóbico aún es un tema pendiente y que falta mucho para ser resuelto aun en países como Estados Unidos (Ortiz y García, 2005). Así, este tipo de discriminación es importante en Latinoamérica; la comunidad LGBTI es una minoría vulnerable a sufrir diversos vejámenes a causa del prejuicio, la exclusión y la falta de educación en la población. Este enfoque negativo respecto orientaciones sexuales diferentes a la heterosexual afecta la vivencia de la homosexualidad como condición, con graves implicaciones en la construcción de la identidad personal y colectiva del adolescente (Cid-Aguayo, Pérez-Villegas y Sáez-Carrillo, 2011; Jocelyn, 2006; Moral de la Rubia, 2011).

De acuerdo con lo anterior, Cooper y Blumenfeld (2012), en una investigación efectuada en Estados Unidos con 310 homosexuales, lesbianas y bisexuales, con edades de entre los 11 y 18 años, buscaron explorar la frecuencia del ciberacoso y su efecto en la vida de los jóvenes. En los resultados se encontró que 56\% de los participantes indicó sentirse deprimidos como resultado de la intimidación cibernética. Además, 35\% indicaron tener pensamientos suicidas como consecuencia de dicha intimidación. Asimismo, se destacó que experimentaron una imagen corporal deficiente $(43 \%)$, aislamiento de amigos $(28 \%)$ y familiares $(27 \%)$, miedo a ir al colegio $(24 \%)$, y bajo rendimiento académico (23\%).

Es importante destacar que los colegios, como espacios de socialización, también se convierten en sitios de amplia convergencia de creencias e imaginarios, siendo lugares de diversidad donde la violencia de género y, en contra de las minorías sexuales, puede ser un fenómeno activo que pasa desapercibido. En este sentido Cénat, Blais, Hébert, Lovoie y Guerrier (2015) desarrollaron en un estudio con 8,194 estudiantes de diferentes instituciones educativas en Quebec, Canadá, en donde las minorías sexuales, en especial homosexuales y bisexuales, eran acosados en el centro (bullying) y por medio de redes sociales. En Colombia, Mura y Diamantini (2013) analizaron una muestra de 360 estudiantes de escuela secundaria (59\% de colegios públicos y $41 \%$ de colegios privados) donde $69 \%$ había sido víctimas de ciberbullying, e indican que no hay distinciones entre estudiantes según el tipo de colegio.

Por esta razón es importante estudiar la situación de la comunidad LGBTI en los colegios frente al fenómeno del ciberbullying, entendiendo las graves consecuencias de la violencia en el desarrollo y salud de las personas victimizadas. También son necesarios más estudios donde se identifique su prevalencia, en ocasiones 
desconocida por la normalización de prácticas discriminadoras o porque sucede en el anonimato, permitiendo que se atiendan las falencias del modelo educativo en la formación para el respeto a los derechos humanos fundamentales y propiciando la aceptación de la diferencia como una característica propia de las sociedades pluriculturales que continúan diversificándose.

Además, a pesar de que el ciberbullying es de interés en algunas investigaciones que exploran la victimización de las minorías sexuales, éstas siguen siendo una población de interés en los trabajos empíricos y de revisión donde se exploren de modo exclusivo aspectos relacionados con este tipo de acoso en jóvenes LGBTI. Por tanto, el objetivo del presente trabajo fue determinar la prevalencia de ciberbullying por homofobia en personas con orientación sexual distinta a la heterosexual, en dos colegios de educación media de la ciudad de Bucaramanga, Colombia.

\section{MÉTodo}

Se efectuó una investigación de tipo cuantitativa, de corte transversal y descriptiva (Hernández, Fernández y Baptista, 2007; Montero y León, 2007), teniendo en cuenta las siguientes variables: 1) ciberbullying; 2) orientación sexual de los participantes, y 3) tipo de colegio (privado y público).

\section{Participantes}

La población estuvo compuesta por 214 estudiantes de educación secundaria de grados octavo $(50 \%)$ y noveno $(50 \%)$ de un colegio público (48\%) y uno privado (52\%), con edades comprendidas entre los 12 y 14 años, de la ciudad de Bucaramanga, Colombia, de los que 101 (53\%) fueron mujeres y 91 (47\%) hombres. Se seleccionó de manera aleatoria un grupo de estudiantes de todos los salones de octavo y noveno, mediante un muestreo no probabilístico por conveniencia (Hernández et al, 2007), aplicando las pruebas sólo quienes tenían consentimiento informado y firmado por el participante $(n=$ 192). Se consideró esta población de estudiantes porque están en la mitad de la adolescencia y de la educación secundaria, momentos críticos del desarrollo y de la etapa educativa en que el bullying y el ciberbullying puede tener más auge, a diferencia de estudiantes de grado décimo y onceavo (Cénat et al., 2015).

\section{Materiales}

Cuestionario sociodemográfico, donde se obtuvo información relacionada con su sexo, orientación sexual, edad, estrato socioeconómico, tiempo que pasaban co- nectados a internet y lugar de su casa donde estaba la computadora con que se conectaban a internet.

Cuestionario Ciberbullying (Garaigordobil y Fernández-Tomé, 2011). No se trata de un instrumento adaptado; es original y tiene un proceso de culturización al ser revisados los ítems con permiso de una de las autoras, sin que hubiera ningún cambio en los mismos. Los coeficientes alfa de Cronbach (ciberagresión $\alpha=$ 0.91; cibervictimización $\alpha=0.82$; ciberobservación $\alpha=$ $0.87)$ evidenciaron una adecuada consistencia interna. Respecto a la validez, correlaciona positiva y significativamente con otras medidas de acoso escolar, y de manera convergente con resolución agresiva de conflictos y conductas antisociales (González, 2015).

Este cuestionario evalúa 15 conductas de ciberbuIlying en tres categorías: víctimas, agresores y observadores. Respecto a las víctimas, permite la identificación de duración del acoso, frecuencia de desarrollo de acciones, edad de los agresores, sentimiento de la víctima, efectos del acoso y acciones emprendidas. Referente a los agresores, indaga acerca de sentimientos experimentados al acosar, identidad utilizada para acosar, razones por las que efectúa el acoso y si la actuación fue grupal o individual. En cuanto a los observadores, identifica pertenencia al grupo acosador, nivel de acuerdo con lo que observa, empatía con las víctimas, acciones que efectúa y sentimientos que tiene al observar dichas conductas. Los ítems se valoran mediante una escala Likert de cuatro puntos $(0=$ nunca; $3=$ siempre $)$.

\section{Procedimiento}

Se consideraron un colegio público y uno privado; se presentó un consentimiento informado a los rectores, donde se solicitaba permiso para efectuar la investigación. Se seleccionó de manera aleatoria la muestra en los grados octavo y noveno, y se procedió a solicitar permiso a los padres de familia por medio del consentimiento informado (de acuerdo con a la Resolución 008430 de 1993 y la Ley 1090 de 2006). En las sesiones se aplicaron a los estudiantes los instrumentos que habían sido autorizados por los padres.

Los cuestionarios se aplicaron durante el horario escolar, indicando a los estudiantes que su participación en el estudio era voluntaria, individual y anónima. Además, los datos fueron procesados con estricta confidencialidad, teniendo en cuenta el anonimato de los participantes, de conformidad con la Ley de protección de datos personales (Ley habeas data), aclarando que no hubo ninguna retribución a los participantes por colaborar en el estudio.

La organización de los datos se hizo en una base de datos de Excel, y para su análisis estadístico se utilizó 
el programa Statistical Package for the Social Sciences (SPSS v.23) en español.

\section{Análisis estadísticos}

El análisis se hizo por medio del paquete estadístico IBMSPSS V21. Se utilizaron las pruebas chi-cuadrado para conocer la relación entre el género y el grado que cursan los estudiantes (tabla 1), análisis de varianzas para determinar si había diferencias estadísticamente significativas entre la conducta promedio de cibervictimización, ciberagresión y ciberobservación entre orientación sexual de manera general y por colegio; además se hizo la prueba a posteriori de Bonferroni en caso que hubiera diferencia.

\section{Resultados}

La distribución de la muestra participante de la investigación, por género y grado, se muestra en la tabla 1.

\section{Tabla 1}

Frecuencia e índice de hombres y mujeres de los grados octavos y noveno.

\begin{tabular}{lccc}
\multicolumn{1}{c}{ Grados } & Hombre & \multicolumn{1}{c}{ MUJer } & TOTAL \\
\hline Octavo & $45(23.4 \%)$ & $51(26.5 \%)$ & $96(100 \%)$ \\
Noveno & $46(23.9 \%)$ & $50(26 \%)$ & $96(100 \%)$ \\
Total & $91(47.3 \%)$ & $101(52.5 \%)$ & \\
Por medio de la prueba Chi-cuadrado de independencia \\
entre las variables, se comprueba que no existe relación \\
estadísticamente significativa entre las variables curso y \\
género $\left(X^{2}=.019 ; p=.891\right)$
\end{tabular}

En la tabla 2 se indican la frecuencia e índices de los estudiantes según su orientación sexual, evidenciando que $5.7 \%$ de los participantes son homosexuales, $4.2 \%$ bisexuales y $90.1 \%$ heterosexuales.

Tabla 2

Frecuencia (índice) de estudiantes según su orientación sexual.

\begin{tabular}{|llll|}
\hline $\begin{array}{c}\text { Orientación } \\
\text { Sexual }\end{array}$ & \multicolumn{1}{c}{ Hombres } & \multicolumn{1}{c|}{ Mujeres } & \multicolumn{1}{c|}{ Total } \\
\hline Homosexuales & $6(3.1 \%)$ & $5(2.6 \%)$ & $11(5.7 \%)$ \\
\hline Bisexuales & $4(2.1 \%)$ & $4(2.1 \%)$ & $8(4.2 \%)$ \\
\hline Heterosexuales & $81(42.1 \%)$ & $92(48 \%)$ & $173(90.1 \%)$ \\
\hline Total & $91(47.3 \%)$ & $101(52.7 \%)$ & $192(100 \%)$ \\
\hline
\end{tabular}

En cuanto a la prevalencia de ciberbullying según la orientación sexual en los colegios estudiados, los resultados mostraron que $27.7 \%$ de los estudiantes homosexuales han sufrido algún tipo de ciberacoso por su orientación, mientras que $25 \%$ de los estudiantes bisexuales manifestaron haber sido víctimas de algún tipo de ciberagresión.

Por otro lado es importante indicar que tanto estudiantes homosexuales como bisexuales también forman parte del grupo de agresores y observadores; así, $72.7 \%$ de los estudiantes homosexuales han sido agresores y $54.4 \%$ han sido observadores, mientras que los estudiantes bisexuales han sido agresores $(50 \%)$ y observadores (75\%). Asimismo, $72.7 \%$ de los estudiantes heterosexuales fueron agresores frentes a $54.4 \%$ que han desempeñado el papel de observadores (tabla 3).

Entre las diferentes orientaciones sexuales se hallaron diferencias estadísticamente significativas en la conducta promedio de cibervictimización y ciberagresión: $(\mathrm{F}(2 ; 187)=5.883 ; \mathrm{p}=0.003)$ y $(\mathrm{F}(2 ; 187)=6.597 ; \mathrm{p}=$ $0.002)$ respectivamente; tal diferencia se presentada entre los heterosexuales y los homosexuales $(p=0.006)$ para el caso de las cibervíctimas, y entre los bisexuales y homosexuales en caso de los ciberagresores ( $p=0.005)$. Por lo contrario, no hubo diferencias significativas para la conducta de la ciberobservación.

\section{Tabla 3}

Frecuencia (índice) de estudiantes homosexuales y bisexuales que han sido víctimas, agresores y observadores.

\begin{tabular}{llll}
$\begin{array}{c}\text { Orientación } \\
\text { sexual }\end{array}$ & \multicolumn{1}{c}{ Víctimas } & Agresores & Observadores \\
\hline Homosexuales & $3(27.7 \%)$ & $8(72.7 \%)$ & $6(54.4 \%)$ \\
Bisexuales & $2(25 \%)$ & $4(50 \%)$ & $6(75 \%)$ \\
Heterosexuales & $69(39.8 \%)$ & $123(71 \%)$ & $64(36.9 \%)$
\end{tabular}

Respecto al tipo de colegio, en la tabla 4 se muestra la frecuencia de estudiantes homosexuales, bisexuales y heterosexuales que han sido tanto víctimas como agresores y observadores. En este sentido, se evidencia un total de cinco estudiantes homosexuales (5\%), dos bisexuales ( $2 \%$ ) y 93 heterosexuales (93\%) en el colegio privado. Por otra parte, en el colegio público se encontraron seis estudiantes homosexuales (6.5\%), seis estudiantes bisexuales $(6.5 \%)$ y 80 estudiantes heterosexuales $(87 \%)$.

Se encontraron diferencias estadísticamente significativas sólo en la conducta de ciberagresión entre el tipo de colegio y preferencia sexual $(F(2 ; 184)=11.55$; $p=0.001$ ), siendo ésta entre los homosexuales y los bisexuales $(p=0.03)$. 


\section{Tabla 4}

Frecuencia (índice) de estudiantes discriminados por colegio, orientación sexual y función que desempeñan en el ciberbullying.

\begin{tabular}{|lllll|}
\hline $\begin{array}{c}\text { Tipo de } \\
\text { Colegio }\end{array}$ & $\begin{array}{c}\text { Orientación } \\
\text { Sexual }\end{array}$ & \multicolumn{1}{c}{ Víctimas } & Agresores & Observadores \\
\hline & Homosexuales & $1(20 \%)$ & $5(100 \%)$ & $3(60 \%)$ \\
\hline $\begin{array}{l}\text { Priva- } \\
\text { do }\end{array}$ & Bisexuales & $\begin{array}{l}2 \\
(100 \%)\end{array}$ & $2(100 \%)$ & $2(100 \%)$ \\
& Heterosexuales & $\begin{array}{l}29 \\
(31.2 \%)\end{array}$ & $\begin{array}{l}77 \\
(82.8 \%)\end{array}$ & $26(27.9 \%)$ \\
\hline & Homosexuales & $\begin{array}{l}2 \\
(33.3 \%)\end{array}$ & $3(50 \%)$ & $3(50 \%)$ \\
\hline $\begin{array}{l}\text { Públi- } \\
\text { co }\end{array}$ & Bisexuales & $0(0 \%)$ & $2(33.3 \%)$ & $4(66.6 \%)$ \\
& Heterosexuales & $\begin{array}{l}40 \\
(50 \%)\end{array}$ & $\begin{array}{l}46 \\
(57.5 \%)\end{array}$ & $38(47.5 \%)$ \\
\hline
\end{tabular}

\section{Discusión}

El objetivo de la presente investigación fue analizar la prevalencia de ciberbullying en estudiantes de secundaria con orientación sexual distinta a la heterosexual de un colegio público y uno privado del área metropolitana de Bucaramanga, es decir, homosexuales y bisexuales, porque las minorías sexuales son víctimas de discriminación y exclusión social en gran medida, aunque también lo son los estudiantes heterosexuales, aspecto que puede explicarse por el auge del uso de las telecomunicaciones y al poco conocimiento que tienen los padres y educadores acerca de las consecuencias del ciberbullying (García-Maldonado et al., 2011; Mura y Diamantini, 2013). Además, en algunos sectores de la sociedad Latinoamericana se presta poca atención a los estereotipos que generan discriminación hacia homosexuales, bisexuales y demás miembros de la comunidad LGBTI (Ortiz y García, 2005; Cid-Aguayo, Pérez-Villegas y Sáez-Carrillo, 2011; Jocelyn, 2006; Moral de la Rubia, 2011), factor que agrava la violencia sexual contra dichas personas.

Respecto a la prevalencia del ciberbullying, en contraste con la investigación de Mura y Diamantini (2013) que indica que $69 \%$ de los estudiantes habían sido víctimas de ciberbullying, los resultados de esta investigación revelan que $38 \%$ de los estudiantes evaluados en algún momento ha sido cibervíctima. Es interesante que los resultados nuestren una mayor prevalencia entre agresores que entre las víctimas, siendo una tendencia significativa que es necesario considerar porque los estudios muestran índices proporcionales de víctimas $(1.5 \%)$ y victimarios (1.7\%) (Ortega, Calmaestra y Mora-Merchán, 2008) o, por lo contrario, donde hay un índice de víctimas más alto $(4.5 \%)$ que el de agresores (3\%) (Avilés, 2009).

Respecto a las diferencias por tipo de colegio, en el privado es evidente que, en su totalidad, $31.6 \%$ ha sido víctima de ciberbullying, aunque $83 \%$ ha sido agresora y $30.6 \%$ ha presenciado este tipo de acoso. Así, en el colegio público se encontró que $46 \%$ de la muestra ha sido alguna vez cibervíctima, $56 \%$ ha incurrido en conductas de ciberagresión y $49 \%$ ha sido observador. Al igual que en el colegio privado, la cantidad de estudiantes que han sido ciberagresores es superior a los demás roles. Son importantes las diferencias entre los colegios público y privado, porque en el primero hay un menor índice de agresores, pero una mayor cantidad de víctimas y observadores. Asimismo, las diferencias estadísticamente significativas se hallaron sólo en la conducta de ciberagresión entre el tipo de colegio y preferencia sexual, siendo ésta entre los homosexuales y los bisexuales. Es posible que haya algunos factores sociodemográficos (geográficos, culturales y educativos) (Garaigordobil, 2011) que estén asociados a mayor prevalencia de cyberbullying en un colegio más que en otro, como la cantidad de horas que el adolescente pasa en redes sociales (como foros o chats), sitios donde puede ser más propenso a esta manera de acoso (Román y Murillo, 2011). Es necesario destacar que la muestra del colegio privado fue con 10 estudiantes más grande que la del colegio público, y que por el tamaño de la muestra pudiera ser significativo.

En cuanto a la orientación sexual, se identificó que los estudiantes con orientación bisexual y heterosexual han participado alguna vez en los tres roles que pueden adoptarse en esta modalidad de acoso. Así, los estudiantes homosexuales han tenido una mayor prevalencia en el ciberbullying como agresores (72.7\%) y como víctimas $(27.7 \%)$, mientras que los estudiantes bisexuales han sido de manera mayoritaria observadores (75\%). Estos resultados se relacionan con los obtenidos por Cénat et al. (2015), que reporta que las minorías sexuales son significativamente acosadas en los centros educativos por medio de redes sociales. Las diferencias estadísticamente significativas se evidenciaron tanto en la cibervictimización como en la ciberagresión entre las diferentes orientaciones sexuales (entre los heterosexuales y los homosexuales para el caso de las cibervíctimas, y entre los bisexuales y homosexuales en caso de los ciberagresores). Por lo contrario, no hubo diferencias significativas respecto a la ciberobservación.

Diferenciando entre el tipo de colegio (público y privado) se encontró que los estudiantes homosexuales son más acosados en los colegios públicos (33.3\%), frente a 
20\% en los privados, así como Cénat et al. (2015) señalan en los resultados de su investigación respecto a que las minorías sexuales son más propensas a ser violentadas por cuestión de identidad sexual. Por lo contrario, referente a los estudiantes homosexuales ciberagresores en el colegio privado, el índice es mucho más alto $(100 \%$ frente a $50 \%$ del colegio público), así como el 50\% que ha participado como observador de estas conductas.

En cuanto a los estudiantes bisexuales, $100 \%$ en el colegio privado ha sido víctima, agresor y observador, mientras que ninguno de los estudiantes del colegio público fue víctima (50\% fue agresor y $50 \%$ observador).

Es importante destacar que estos resultados son muy importantes porque estas formas más graves de agresión están en línea con los resultados hallados por Elipe, De la Oliva y Del Rey (2018) respecto a que la orientación sexual y de identidad son los principales motivos en los crímenes por odio.

\section{Conclusiones}

Los resultados indican que las minorías sexuales son víctimas de ciberbullying en colegios públicos y privados, y que los índices de ciberacoso varían de acuerdo con el colegio donde éste suceda. En este caso, el colegio privado presentó datos significativos de ciberacoso generalizado, es decir, sin distinción de la condición sexual, en comparación del público. No obstante, el colegio público también muestra índices que indican que esta modalidad de acoso es prevalente entre la comunidad educativa, sobre todo contra las minorías sexuales. Es necesario educar para la inclusión en los colegios colombianos; evitar la violencia entre pares permite el establecimiento de medio de socialización basados en la paz, el diálogo y la comprensión mutua.

Es importante combatir y erradicar la homofobia; las instituciones deben establecer acciones urgentes que incidan en un cambio del modelo cultural que ha implantado la heterosexualidad como única manera válida en el ejercicio del amor y la sexualidad, porque el heterosexismo intenso y persistente de la sociedad contemporánea se ha implantado como sistema dominante y conductor de las relaciones, imponiendo de por sí una heteronormatividad, que obliga a que surja, por antonomasia, la homofobia (García, s. f.; Mercado, 2009; Ríos, 2011).

Además se recomiendan nuevas investigaciones con muestras más amplias en Bucaramanga y su área metropolitana, así como en otras ciudades de Colombia, para lograr mayor objetividad en el diagnóstico de la prevalencia del ciberbullying contra las minorías sexuales. Los datos presentados aquí son apenas un indicador de la realidad novedosa que las TIC ofrecen en la población joven colombiana, que exige otras maneras de abordar diferentes problemáticas.

En cuanto a las limitaciones de este estudio, sobre todo el uso de cuestionarios de autoinforme, junto con la resistencia de los participantes a reconocer su orientación sexual, así como el muestreo utilizado, no permite la generalización de los resultados. Sin embargo, la naturaleza de los fenómenos tratados, en particular la victimización en minorías sexuales, hace difícil trabajar con grupos de personas lo suficientemente grandes y enfocarse en cada uno de ellos. En cualquier caso, es fundamental continuar investigando y visibilizando una realidad que, debido a su invisibilidad, no es fácil de abordar.

\section{Referencias}

Acosta, M., Cuellar, L., \& Martínez, L. (2013). Colombia: El bullying por homofobia debe salir del clóset. Bogotá, Washington D.C.: Banco Interamericano de Desarrollo (BID).

Arseneault, L., Bowes, L., \& Shakoor, S. (2010). Bullying victimization in youths and mental health problems: "Much ado about nothing." Psychological Medicine, 40, 717-729. doi:10.1017/S0033291709991383

Artiles, A. \& Kozleski, E. (2014). Educación inclusiva en el siglo XXI: Notas para un programa de investigación históricocultural. En M.C. Cardona \& E. Chiner (eds.). Investigación educativa en escenarios diversos, plurales y globales (pp. 51-65). Alicante: AIDIPE.

Avilés, J. (2009). Ciberbullying, diferencias entre el alumnado de secundaria. Boletín de Psicología, 96, 79-96. Recuperado de https://www.uv.es/seoane/boletin/previos/N96-6.pdf

Besley, B. (2005). Ciberbullying: An emerging threat to the always on generation. Retrieved from http://www.ciberbullying. $\mathrm{ca} / \mathrm{pdf} /$ Ciberbullying Article by Bill Belsey.pdf

Blair, E. (2009). Aproximación teórica al concepto de violencia: Avatares de una definición. Política y Cultura, 32, 9-33. Recuperado de http://www.scielo.org.mx/pdf/polcul/n32/ n32a2.pdf

Cénat, J., Blais, M., Hébert, M., Lovoie, F., \& Guerrier, M. (2015). Correlates of bullying in Quebec high shool students: The vulnerability of sexual-minority youth. Journal of affective disorders, 183, 315-321. https://doi.org/10.1016/j. jad.2015.05.011

Chaux, E. (2012). Educación, convivencia y agresión escolar. Bogotá: Universidad de los Andes.

Cid-Aguayo, M., Pérez-Villegas, R., \& Sáez- Carrillo, K. (2011). Orientación sexual en los adolescentes y su asociación con variables demográficas, socioconductuales y sexuales. Perinatología y Reproducción Humana, 25(2), 74-80. Recuperado de https://www.medigraphic.com/ pdfs/inper/ip-2011/ip112c.pdf

Cooper, R.M. \& Blumenfeld, W.J. (2012). Responses to Ciberbullying: A descriptive analysis of the frequency of and impact on LGBT and allied youth. Journal of LGBT Youth, 9(2), 153-177. doi: 10.1080/19361653.2011.649616 
Cruz, S. (2002). Homofobia y masculinidad. El Cotidiano, 18(113), 8-14. Recuperado de https://www.redalyc.org/ pdf/325/32511302.pdf

Elipe, P., De la Oliva, M., \& Del Rey, R. (2018). Homophobic bullying and ciberbullying: Study of a silenced problem. Journal of homosexuality, 65(5), 672-686. https://doi.org/ 10.1080/00918369.2017.1333809

Espejo, J. C. (2018). Discriminación y violencia homofóbica en el sistema escolar: estrategias de prevención, manejo y combate. Revista Brasileira de Educação, 23, 1-24. http:// dx.doi.org/10.1590/s1413-24782018230031

Espelage, D. L., Low, S., Van Ryzin, M. J., \& Polanin, J. R. (2015). Clinical trial of second step middle school program: Impact on bullying, ciberbullying, homophobic teasing, and sexual harassment perpetration. School Psychology Review, 44(4), 464-479. https://doi.org/10.1016/j. appdev.2014.11.007

Finkelhor, D., Mitchell, K., \& Wolak, J. (2000). Online victimization: A reporto on the nation's youth. National center for missing \& exploited children all rights reserved. Retrieved from http://www.unh.edu/ccrc/pdf/Victimization_Online_ Survey.pdf

Garaigordobil, M. (2011). Prevalencia y consecuencias del ciberbullying: Una revisión. International Journal of Psychology and Psychological Therapy, 11(2), 233-254. Recuperado de https://www.redalyc.org/ pdf/560/56019292003.pdf

Garaigordobil, M., \& Fernández-Tomé, A. (2011). Cuestionario de Ciberbullying. Recuperado de http://www. psicologiaysexologia.org/wp-content/uploads/2013/11/ Bullying-y-cyberbullyung.pdf

García-Maldonado, G., Joffre-Velazquez, V., Martínez-Salazar, G., \& Llanes-Castillo, A. (2011). Ciberbullying: Forma virtual de intimidación escolar. Revista colombiana de psiquiatría, 40(1), 115-130. https://doi.org/10.1016/ S0034-7450(14)60108-6

García, J. (s.f.). La discriminación por motivo de orientación sexual e identidad de género. Homofobia, transfobia y derechos humanos. Laboratorio de sociología jurídica. Universidad de Zaragoza. Recuperado de http://www. tiempodelosderechos.es/docs/may12/homofobia.pdf

González, B. (2015). Los observadores ante el ciberacoso (cyberbullying). Revista Investigación en la Escuela, 87, 81-90. Recuperado de https://idus.us.es/ xmlui/bitstream/handle/11441/59696/R87-6. pdf? sequence $=1$ \&isAllowed $=y$

Grigg, D.W. (2010). Cyber-aggression: Definition and concept of ciberbullying. Australian Journal of Guidance and Counseling, 20(2), 143-156. doi: https://doi.org/10.1375/ ajgc.20.2.143

Hernández, R., Fernández, C., \& Baptista, P. (2007). Metodología de la investigación. México D.F: Mc Graw Hill.

Hernández, P., \& Solano, I. (2007). Ciberbullying, un problema de acoso escolar. Revista Iberoamericana de Educación a Distancia, 10(1), 17-36. Recuperado de https://digitum. um.es/digitum/bitstream/10201/14613/1/ciberbullyng_ Hernandez_Solano_2007.pdf

Jocelyn, P. (2006). Construcción de la identidad sexual en adolescentes hombres y mujeres de 13 a 18 años en
Castro, Chiloé. (Tesis de pregrado). Universidad Austral de Chile. Valdivia: Chile.

Keith, S. \& Martin, M.E. (2005). Cyber-Bullying: Creating a culture of respect in a cyber world. Reclaiming Children and Youth, 13, 224-228. Retrieved from https://bienestaryproteccioninfantil.es/imagenes/ tablaContenidos03SubSec/13 4 Keith Martin.pdf

Lorenzo, M. (2012). Nuevas formas de violencia entre pares: Del bullying al cyberbuying. Revista Médica del Uruguay, 28(1), 48-53. Recuperado de http://www.scielo.edu.uy/ $\mathrm{pdf} / \mathrm{rmu} / \mathrm{v} 28 \mathrm{n} 1 / \mathrm{v} 28 \mathrm{n} 1 \mathrm{a} 07 . \mathrm{pdf}$

Mercado, J. (2009). Intolerancia a la diversidad sexual y crímenes por homofobia. Un análisis sociológico. Sociológica, 24(69), 123-156. Recuperado de http://www.scielo.org. $\mathrm{mx} / \mathrm{pdf} / \mathrm{soc} / \mathrm{v} 24 \mathrm{n} 69 / \mathrm{v} 24 \mathrm{n} 69 \mathrm{a} 7 . \mathrm{pdf}$

Montero, I., \& León, O. (2007). A guide for naming research studies in psychology. International Journal of Clinical and Health Psychology, 7(3), 847-862. Recuperado de https:// www.redalyc.org/pdf/337/33770318.pdf

Moral de la Rubia, J. (2011). Orientación sexual en adolescentes y jóvenes mexicanos de 12 a 29 años. Psicología desde el Caribe, 1(27), 112-135. Recuperado de https://www. redalyc.org/pdf/213/21320708006.pdf

Morales, W. F., Rueda, Á. Y., Redondo, J., Luzardo, M. \& Gómez, N. (2018). Factores personales, de género y socioeconómicos relacionados con el ciberbullying en adolescentes colombianos. Revista Digital Internacional de Psicología y Ciencia Social, 4(1), 46-62. https://doi. org/10.22402/j.rdipycs.unam.4.1.2018.153.46-62

Mura, G., \& Diamantini, D. (2013). Ciberbullying among Colombian students: An exploratory investigation. European journal of investigation in health, Psychology an Education, 3(3), 249-256. doi: 10.1989/ejihpe.v3i3.47.

Oliveros, M., Amemiya, I., Condorimay, Y., Oliveros, R., Barrientos, A., \& Rivas, B. E. (2012). Ciberbullying: Nueva tecnología electrónica al servicio del acoso escolar en alumnos de dos distritos de Lima, Perú. Anales de la Facultad de Medicina, 73(1), 13-18. Recuperado de http:// www.scielo.org.pe/pdf/afm/v73n1/a03v73n1.pdf

Ortega R., CalmaestraJ., \& Mora-Merchán,J. (2008). Ciberbullying. International Journal of Psychology and Psychological Therapy, 8, 183-192. Recuperado de https://idus.us.es/ xmlui/bitstream/handle/11441/58864/cyberbullying\%20 $\% 281 \% 29 . p d f$ ? sequence $=1$ \&isAllowed $=y$

Ortiz, L., \& García, M. (2005). Efecto de la violencia y la discriminación en la salud mental de bisexuales, lesbianas y homosexuales de la ciudad de México. Cadernos de saúde pública, 21(3), 913-925. doi: 10.1590/S0102$311 \times 2005000300026$

Personería Municipal de Santiago de Cali. (2012). Conviviendo con las diferencias educación para la paz y los derechos. Guía \# 1. Para la Prevención y atención integral del acoso escolar. Recuperado de http://web1.cali.gov.co/ educacion/publicaciones/descargar.php?id=40796

Piedra, J., Rembrandt, A., Ries, F., \& Ramírez, G. (2013). Homofobia, heterosexismo y educación física: Percepciones del alumnado. Profesorado. Revista de Currículum y Formación del Profesorado, 17(1), 325-338. Recuperado de http://www.redalyc.org/articulo.oa?id=56726350020 
Redondo, J., Luzardo, M., \& Rangel, K. (2016). Ciberagresión: Un estudio sobre la prevalencia en estudiantes universitarios colombianos. Informes Psicológicos, 16(1), 85-99. doi: http://dx.doi.org/10.18566/infpsicv16n1a05

Ríos, J. (2011). La homofobia en las escuelas: Implicaciones para desarrollo del líder LGBT (Tesis doctoral). Universidad del Turabo, Gurabo, Puerto Rico.

Rojas, L. (1996). Las semillas de la violencia. Barcelona, España: Espasa Libros.

Román, M., \& Murillo, J. (2011). América Latina: Violencia entre estudiantes y desempeño escolar. Cepal, 104(1), 3754. Recuperado de http://www.eclac.org/publicaciones/ $\mathrm{xml} / 3 / 44073 / \mathrm{RVE} 104$ RomanMurillo.pdf

Simkin, H., \& Becerra, H. (2013). El proceso de la socialización. Apuntes para su exploración en el campo psicosocial. Ciencia, Docencia y Tecnología, 24(47), 119-142. Recuperado de https://www.redalyc.org/ pdf/145/14529884005.pdf
Smith, P.K. (2000). What good schools can do about bullying. Chilhood, 7, 193-212. https://doi. org/10.1177/0907568200007002005

Tucker, J. (2009). Social network has hidden dangers for teens, San Francisco Chronicle, Retrieved from http://www. sfgate.com/news/article/Social-networking-has-hiddendangersfor-teens-3289805.php

UNESCO. (2013). Education sector responses to homophobic bullying. París, France: UNESCO. Retrieved from http:// unesdoc.unesco.org/images/0022/002229/222918S.pdf

Williams, K., \& Guerra, N. (2007). Prevalence and predictors of internet bullying. Journal of Adolescent Health, 41, 14-21. https://doi.org/10.1016/j.jadohealth.2007.08.018 


\section{Meta-Análisis del Artículo}




\section{Dimensión Cuantitativa}

\section{Perfil de Evaluación entre pares}
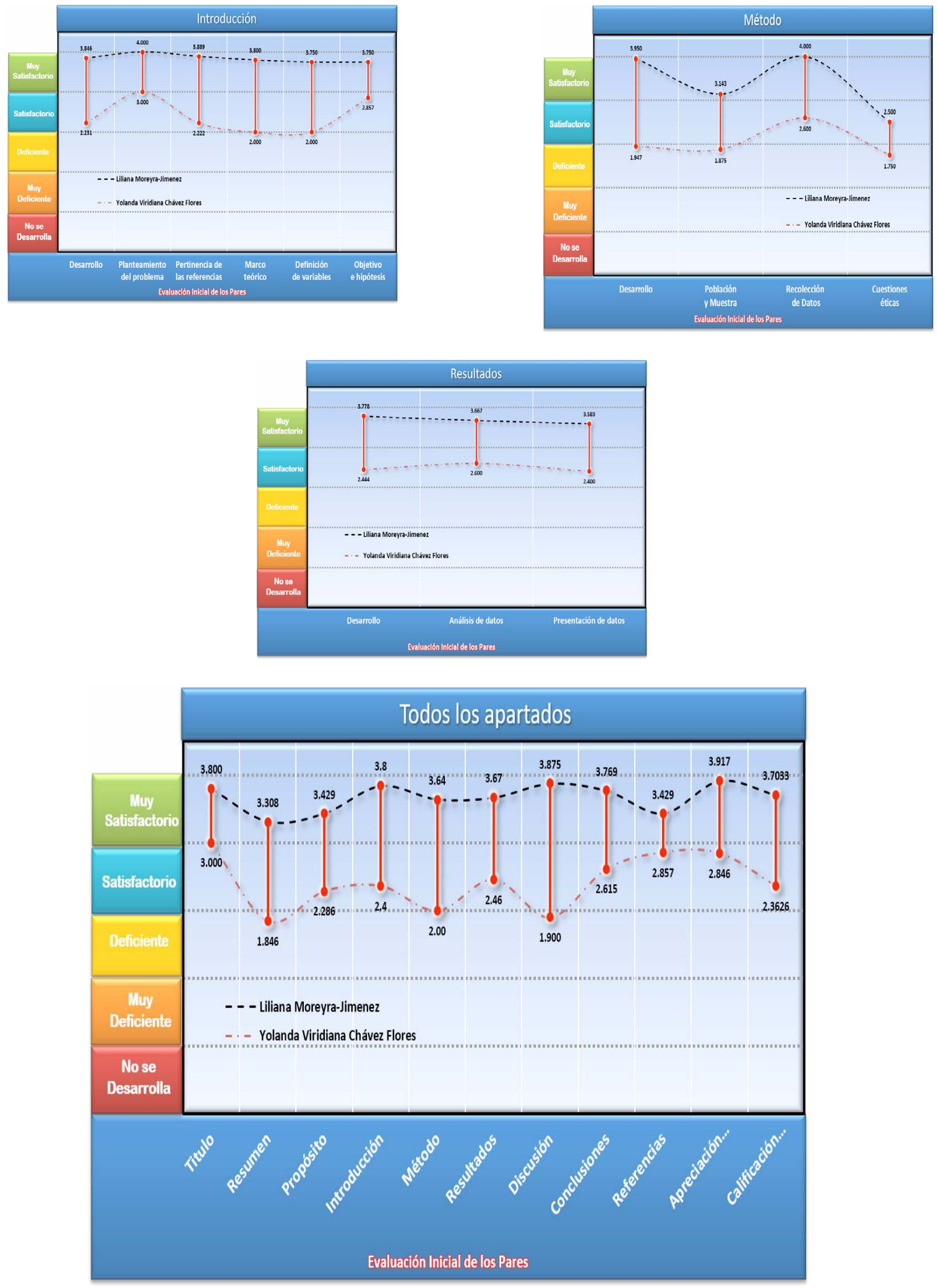


\section{Índice de Concordancia}

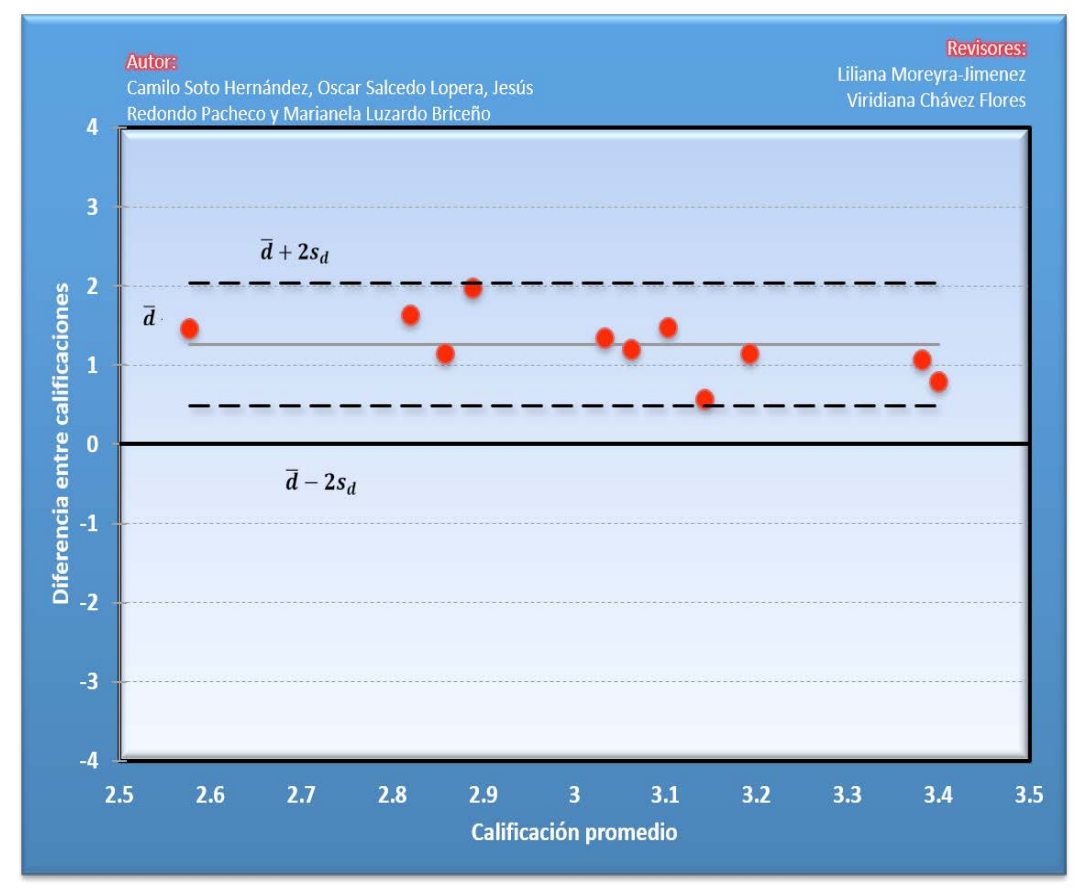

Índice de Acuerdo

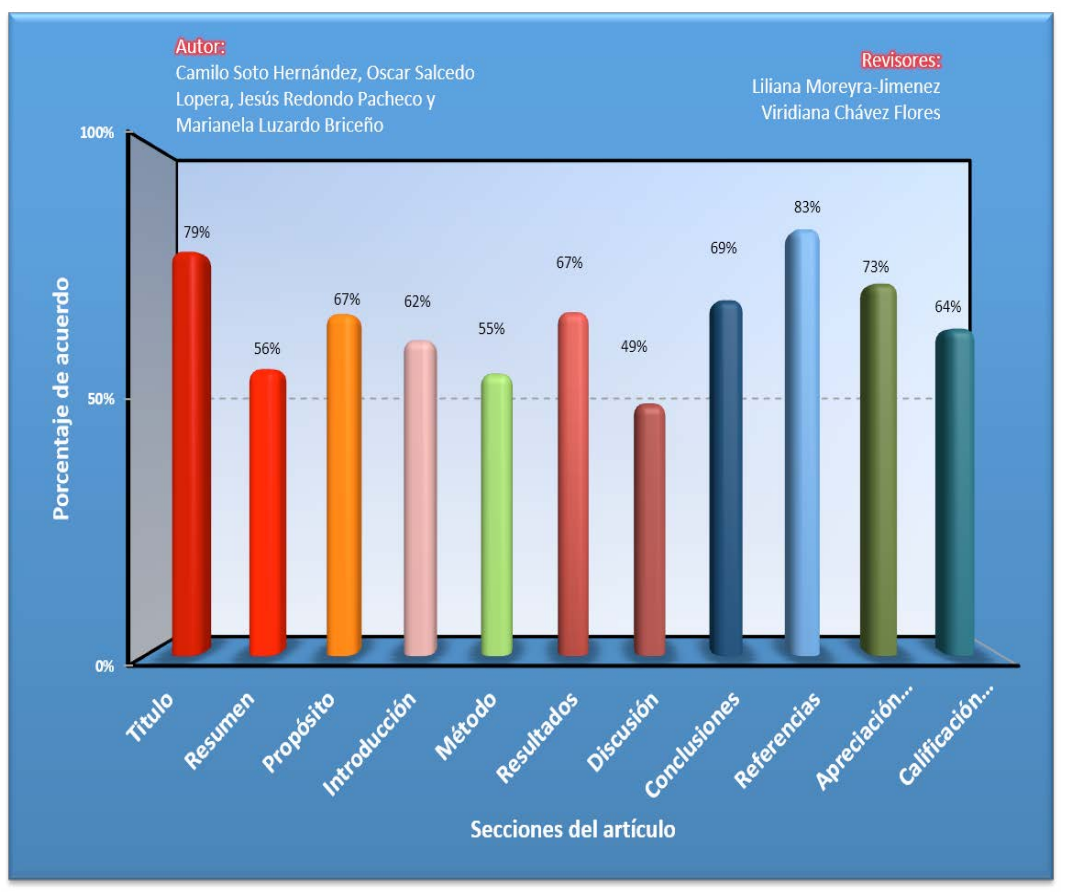




\section{Dimensión Cualitativa}

\begin{tabular}{|c|c|}
\hline Revisor 1 & Revisor 2 \\
\hline Liliana Moreyra Jiménez & Yolanda Viridiana Chávez Flores \\
\hline \multicolumn{2}{|c|}{ Título/Autoría } \\
\hline $\begin{array}{l}\text { Para mejorar el título se sugiere eliminar la palabra "les- } \\
\text { bianas", esto debido a que en los resultados de la investiga- } \\
\text { ción sólo se obtienen datos de estudiantes con orientación } \\
\text { sexual homosexual y bisexual. }\end{array}$ & $\begin{array}{l}\text { Sí se incluyeron los grados académicos de los autores. El } \\
\text { título son } 21 \text { palabras. Sugiero describir a la muestra como } \\
\text { estudiantes colombianos homosexuales y bisexuales o } \\
\text { como minorías sexuales. Sugiero precisar si son adoles- } \\
\text { centes o jóvenes, más que el grado escolar, pues la edad y } \\
\text { el sexo son variables más relevantes. }\end{array}$ \\
\hline \multicolumn{2}{|c|}{ Resumen } \\
\hline $\begin{array}{l}\text { El resumen puede mejorar si se describe la metodolo- } \\
\text { gía/diseño de la investigación. }\end{array}$ & $\begin{array}{l}\text {-Se dedica mucho espacio a la introducción y se acorta el } \\
\text { resto de las secciones. Además, se puede prescindir de la } \\
\text { mayor parte de la información introductoria. -Tal vez el } \\
\text { objetivo sea determinar la prevalencia. Dice determinar } \\
\text { la existencia de cyberbullying e identificar su prevalencia, } \\
\text { pero suena redundante. - Falta agregar el desarrollo del } \\
\text { método y describir a los participantes. - No generalizar los } \\
\text { resultados mencionados en el resumen, pues la muestra no } \\
\text { fue aleatoria y especificar que los resultados se aplican solo } \\
\text { en los colegios analizados. -Precisar el \% de prevalencia en- } \\
\text { contrado, pues solo dice alta prevalencia. -Concluyen que } \\
\text { no se pudo establecer si el cyberbullying al que estuvieron } \\
\text { expuestos fue por razón de su orientación sexual, pero ese } \\
\text { no era el objetivo. -Las palabras clave seleccionarlas de un } \\
\text { tesauro. Público y privado no son buenos descriptores del } \\
\text { estudio. -Revisar la traducción al inglés del resumen. }\end{array}$ \\
\hline \multicolumn{2}{|c|}{ Próposito del Estudio } \\
\hline No tengo comentarios en está sección. & $\begin{array}{l}\text { Me parece que el tema es de suma relevancia, sin embar- } \\
\text { go considero que no se ha revisado la congruencia entre } \\
\text { el objetivo, los análisis, resultados y discusión a la que se } \\
\text { llega. Se da mucha importancia al tipo de colegio (pú- } \\
\text { blico o privado) pero creo que seria importante analizar } \\
\text { también el sexo y la edad. Además de otras variables que } \\
\text { tomaron del cuestionario sociodemográfico. El propósi- } \\
\text { to no se deriva de la literatura señalada. }\end{array}$ \\
\hline
\end{tabular}




\begin{tabular}{|c|c|}
\hline Revisor 1 & Revisor 2 \\
\hline \multicolumn{2}{|c|}{ Introducción } \\
\hline No tengo comentarios para la introducción. & $\begin{array}{l}\text { Me parece que en la introducción se hacen juicios de va- } \\
\text { lor, por ejemplo: son varias generaciones las que han cre- } \\
\text { cido en un contexto violento sin tener una idea concisa } \\
\text { de lo que es vivir en un ambiente de paz. -Me parece que } \\
\text { sería mas adecuado iniciar la introducción con la defini- } \\
\text { ción de las variables de estudio de manera directa al tema. } \\
\text { - Considero que el inicio de la introducción es el párrafo } \\
\text { que inicia con: la incursión de las nuevas tecnologías... } \\
\text { pues ahí es donde se comienza a abordar el tema de inte- } \\
\text { rés. - Se define violencia, pero sería más adecuado definir } \\
\text { bullying o cyberbullying. En este sentido, creo se utlizan } \\
\text { como sinónimos acoso, violencia, cyberbullying lo que es } \\
\text { incorrecto, pues aunque pueden estar asociados, implican } \\
\text { marco teórico distinto, - En la descripción que se hace de } \\
\text { antecedentes, no se indica la edad y el tipo de muestra en } \\
\text { que se hicieron los estudios. además se refieren a datos } \\
\text { de prevalencia como "otros" "alta prevalencia" "escasos } \\
\text { estudios" y se deben mencionar los datos precisos. -pá- } \\
\text { gina } 12 \text { Dice:" el cyberbullying como forma de violencia } \\
\text { puede basarse en cualquier pretexto para ser efectuado". } \\
\text { Pienso que es más complejo que un pretexto. Mas bien } \\
\text { hacer énfasis en la relación del cyberbullying con otras } \\
\text { variables, según antecedentes. -Pag. 12: "Es importante } \\
\text { combatir y erradicar la homofobia, las instituciones de- } \\
\text { ben tomar acciones urgentes que incidan en un cambio } \\
\text { del patrón cultural" En la introducción no es adecuado } \\
\text { hacer recomendaciones o conclusiones. -Definir LGBTI } \\
\text { antes de resumirla. -Pag. 13, se muestra otro juicio de va- } \\
\text { lor: en pleno siglo XXI se siguen presentando este tipo } \\
\text { de eventos desafortunados y reprochables entre la comu- } \\
\text { nidad estudiantil. - Sería mejor buscar antecedentes de } \\
\text { ciberbullying, pues discriminación es otra variable. -Falta } \\
\text { justificar mejor la relevancia del estudio y cerrar la intro- } \\
\text { ducción con el objetivo de investigación o la pregunta. }\end{array}$ \\
\hline
\end{tabular}




\begin{tabular}{|c|c|}
\hline Revisor 1 & Revisor 2 \\
\hline \multicolumn{2}{|c|}{ Método } \\
\hline $\begin{array}{l}\text { Es recomendable incluir la forma en que se aseguró la } \\
\text { confidencialidad de los participantes en el estudio. }\end{array}$ & $\begin{array}{l}\text { - Falta precisar Cuantas secundarias? Solo una publica y } \\
\text { una privada? De qué edades? -Cuáles fueron los criterios de } \\
\text { inclusión y de exclusión? -Cuál fue la técnica de muestreo? } \\
\text { El grupo de estudiantes de cada salón, cómo se determi- } \\
\text { nó el tamaño? O fue por conveniencia? - Aclarar cuantos } \\
\text { alumnos eran en total, cuantos invitaron, cuantos se eva- } \\
\text { luaron y cuantos se incluyeron en los análisis. -Las variables: } \\
\text { el tiempo que pasaban conectados a internet, el lugar de su } \\
\text { casa donde se encontraba el computador del cual se conec- } \\
\text { taban a internet, era importante para los resultados de inte- } \\
\text { rés? Sin embargo, se preguntó también la edad, sexo, nivel } \\
\text { socioeconómico, que sí son relevantes para incluirse en el } \\
\text { estudio. Esta información debería reportarse en la tabla } 1 . \\
\text {-Falta mayor información sobre el cuestionario. ¿Quién lo } \\
\text { realizo? ¿Esta validado para población de Colombia? ¿Qué } \\
\text { opciones de respuesta tiene? ¿Cómo se califica? ¿Qué otra } \\
\text { evidencia de sus propiedades psicométricas existe? ¿Se le ha } \\
\text { hecho un análisis factorial confirmatorio como evidencia } \\
\text { de validez de constructo? - ¿Qué consideraciones éticas se } \\
\text { hicieron? Dado el tema estudiado, es de suma relevancia. } \\
\text { ¿Fue anónimo? ¿Confidencial? -Donde dice mediciones, } \\
\text { es diseño del estudio. - Pag. 17: Se utilizaron las pruebas } \\
\text { chi-cuadrado para conocer la relación entre el género y el } \\
\text { grado que cursan los estudiantes. Pero esa información no } \\
\text { era parte del objetivo. En todo caso sería de interés hacer } \\
\text { el análisis de prevalencia de cyberbullying por género, por } \\
\text { edad o por tipo de colegio. - }\end{array}$ \\
\hline \multicolumn{2}{|r|}{ Resultados } \\
\hline No existen comentarios para la sección de resultados. & $\begin{array}{l}\text {-Pag. 18. Dice: tipo de acoso por su orientación sexual. La } \\
\text { variable de interés es cybercullying. Sugiero revisar el texto } \\
\text { y no utilizar como sinónimo el acoso, discriminación, vio- } \\
\text { lencia, etc. - ¿Hubo diferencias estadísticamente significa- } \\
\text { tivas entre el tipo de orientación sexual y el ser víctima de } \\
\text { cyberbulling? -Tabla 4: ¿hubo diferencias significativas en- } \\
\text { tre los grupos? describir en los resultados: qué se encontró? } \\
\text { Hay o no diferencias en las prevalencias de cyberbullying } \\
\text { entre tipo de colegio, orientación sexual o el papel que des- } \\
\text { empeñaron? -Sugiero trabajar en el formato de las tablas. } \\
\text { Hacer otra columna en lugar de poner los datos abajo. Ta- } \\
\text { bla } 1 \text {. Dar mayor información sobre los participantes. -Creo } \\
\text { que los análisis realizados no son suficientes para describir } \\
\text { el fenómeno estudiado, según su objetivo. }\end{array}$ \\
\hline
\end{tabular}


Revisor 1

Revisor 2

\section{Discusión}

No existen comentarios para la sección de discusión.

\section{Conclusiones}

-Sugiero Iniciar por el objetivo del estudio. Precisar la prevalencia, que era el objetivo general. -Pag. 21. La última

No existen comentarios para la sección de conclusiones. línea, es un error de redacción, ¿̇ se refiere a la violencia ejercida por padre y educadores? - No hay limitaciones, implicaciones de los resultados.

\section{Referencias}

Recomiendo revisar el orden de la lista de referencias. Sugiero agregar el DOI a cada artículo incluido. 


\section{Historia del Proceso}

EdITORIAL

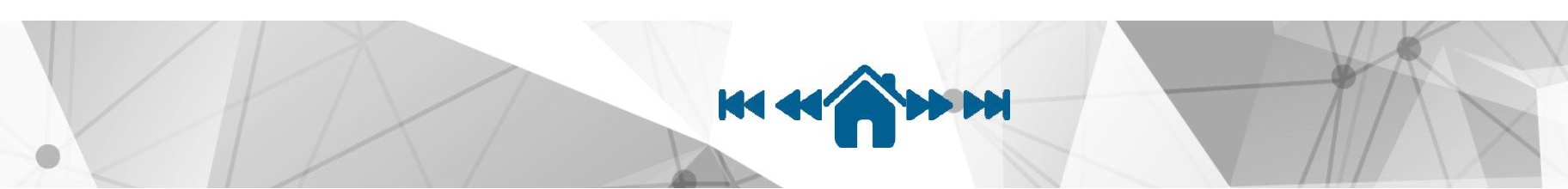

